\title{
Cooperative frequency control with a multi-terminal high-voltage DC network *
}

\author{
Alain Sarlette $^{\text {a }}$, Jing Dai ${ }^{\text {b }}$, Yannick Phulpin ${ }^{\mathrm{c}}$, Damien Ernst ${ }^{\mathrm{d}}$ \\ ${ }^{a}$ SYSTeMS, Ghent University, Technologiepark Zwijnaarde 914, 9052 Zwijnaarde, Belgium \\ ${ }^{\mathrm{b}}$ Department of Power and Energy Systems, SUPELEC, 3 rue Joliot Curie, 91192 Gif-sur-Yvette, France \\ 'INESC Porto, FEUP campus, Rua Dr. Roberto Frias 378, 4200 - 465 Porto, Portugal \\ ${ }^{\mathrm{d}}$ Department of Electrical Engineering and Computer Science, University of Liège, B-4000 Liège, Belgium
}

\begin{abstract}
We consider frequency control in power systems made of several non-synchronous AC areas connected by a multi-terminal high-voltage direct current (HVDC) grid. We propose two HVDC control schemes to make the areas collectively react to power imbalances, so that individual areas can schedule smaller power reserves. The first scheme modifies the power injected by each area into the DC grid as a function of frequency deviations of neighboring AC areas. The second scheme changes the DC voltage of each converter as a function of its own area's frequency only, relying on the physical network to obtain a collective reaction. For both schemes, we prove convergence of the closed-loop system with heterogeneous AC areas.
\end{abstract}

Key words: power system control, frequency control, HVDC systems, decentralized control.

\section{Introduction}

During the last decades, the dynamical systems and control literature has investigated a variety of mechanisms to induce and exploit cooperation in networks. Electrical power networks are a prominent application domain where cooperative reactions allow substantial savings. Probably the most well-known cooperative reaction mechanism in power networks is the so-called primary frequency control [15], whose aim is to counter imbalances between power consumption (or 'load') and generation at short timescales in an alternating current (AC) network. It exploits the fact that any imbalance induces variations of the common frequency throughout the entire network [8], so all the network's units participating

\footnotetext{
* This paper has not been presented at any IFAC meeting. It presents research results of the Belgian Network DYSCO (Dynamical Systems, Control, and Optimization), funded by the Interuniversity Attraction Poles Programme, initiated by the Belgian State, Science Policy Office. Corresponding author J. Dai. Tel. +33675704214.

Email addresses: alain.sarlette@ugent.be (Alain Sarlette), jing.dai@supelec.fr (Jing Dai), email@yphulpin.eu (Yannick Phulpin), dernst@ulg.ac.be (Damien Ernst).
}

in primary frequency control can sense even remote [unknown] power imbalances through [measured] frequency deviations and adapt their effort to correct them. Since the efforts of these units sum up, large synchronous areas can achieve economies of scale $[2,14]$. This has been one of the motivations for e.g. interconnecting regional and national systems into the synchronous grid of Continental Europe, supplying over 400 million customers in 24 countries.

The trend of interconnecting AC systems into larger networks is still ongoing. However, engineers now favor a direct current (DC) technology instead of AC links for the interconnections. This leads among others to lower electrical losses and no need for reactive compensation in submarine and underground transmission links. In a network resulting from interconnection with such a highvoltage direct current (HVDC) system — see Figure 1 - each AC subnetwork (area) is linked to a terminal of a DC grid through a controlled power electronic device (converter), which can set for instance its DC-side voltage or the power flow that it transmits. The effective coupling between the $\mathrm{AC}$ areas then depends on the converters' algorithms. In particular, since the AC areas are not directly interconnected, they are not physically restricted to have identical nor even correlated frequen- 


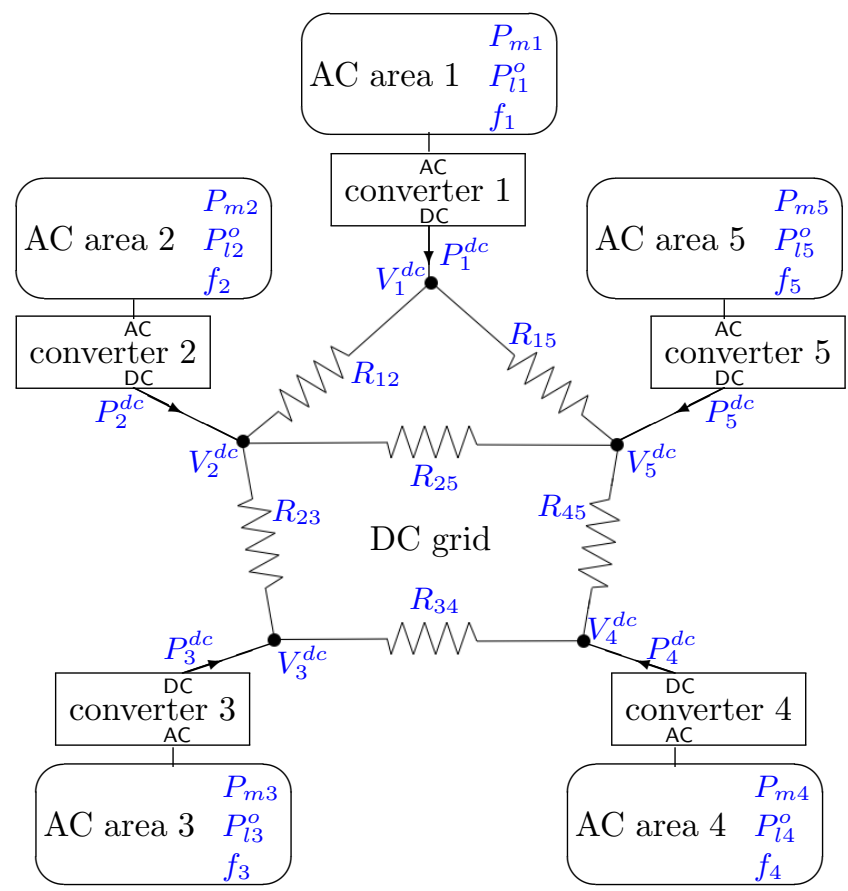

Fig. 1. Multi-terminal HVDC system connecting five AC areas via converters. Variables are explained in Section 2. Measured outputs are the frequencies $f_{i}$ in AC areas $i=1,2, \ldots$. The scalar control input at each converter $i$ is selected from either $P_{i}^{d c}$ (power injection) or $V_{i}^{d c}$ (DC-side voltage).

cies. On one hand, this offers the possibility to use the DC links as safety barriers to prevent faulty $\mathrm{AC}$ subnetworks from harming healthy ones. But on the other hand, it means that even in a healthy network, the units participating in primary frequency control will not necessarily respond to and help counteract imbalances located in remote AC areas. Precisely the current HVDC operating practice, with converters transferring a scheduled amount of power regardless of AC area states, implies independent frequency dynamics in the different $\mathrm{AC}$ areas, which leads to no imbalance sharing between them. To recover imbalance sharing between the different $\mathrm{AC}$ areas, the HVDC system requires specifically designed control algorithms, that let the converters react to current AC area states. The modern HVDC converters' quick actuation capabilities make this a realistic goal.

Recently, several researchers have sought to extend the real-time collective reaction of single-area $\mathrm{AC}$ systems to systems made of several AC areas connected by an HVDC grid. Converter control algorithms have been proposed for the special case of two AC areas, regulating the power exchanged on their single link, see e.g. [1,7,9,18 $20]$. In the present paper, we propose and analyze two cooperative primary frequency control algorithms for $a r$ bitrary networks, with power or voltage steering at the converters. Both algorithms are designed on the basis of cooperative decentralized control. Their explicit target is to drive the frequency deviations of all areas to- wards a common value, mimicking the collective reaction in a single AC network. This target also allows us to exploit the so-called consensus viewpoint from the distributed control literature for algorithm design, see e.g. $[6,11,16,21]$. The first controller adapts the power injections from each $\mathrm{AC}$ area converter into the DC grid as a function of neighboring areas' frequency deviations, with a proportional-integral consensus type action. Coordination among local controllers is ensured by communicating frequency values across the network. This leads to a generalization of the two-area control laws from the literature. In our second controller, each converter reacts to frequency deviations in its own AC area only; but, instead of acting on power injections, it adjusts the voltage of the DC grid node. This "signals" the area's needs as it affects power flows throughout the network. The physical coupling in the DC grid then induces a cooperative behavior, without requiring any explicit communication. This appears to be an original control strategy. We prove that both algorithms yield a stable overall dynamical system with favorable imbalance sharing. Notably, our analysis covers the realistic case where all subsystems can be different. Simulations on a system with five AC areas illustrate the controllers' effectiveness. We have presented the ideas behind this work to power systems researchers, see [3-5]. The goal of this paper is to provide (i) a comprehensive decentralized control viewpoint on the algorithms; and (ii) a new theoretical study of the equilibria and their stability, holding for non-identical subsystems.

The paper is organized as follows. Section 2 gives a mathematical model of multi-terminal HVDC systems. Sections 3 and 4 describe and analyze the two control schemes. Section 5 presents simulation results.

\section{Multi-terminal HVDC system model}

The multi-terminal HVDC system is composed of a DC grid, $N$ separate AC areas, and $N$ converters that interface the AC areas with the DC grid (see Figure 1).

Each AC area $i$, for $i=1,2, \ldots, N$, has a state vector $\left(f_{i}, P_{m i}\right) \in \mathbb{R}^{2}$ and is governed by

$$
\begin{aligned}
J_{i} \frac{d}{d t} f_{i} & =\frac{P_{m i}-P_{l i}-P_{i}^{d c}}{4 \pi^{2} f_{i}}-D_{g i}\left(f_{i}-f_{\text {nom }, i}\right) \\
T_{s m i} \frac{d}{d t} P_{m i} & =P_{m i}^{o}-P_{m i}-\frac{P_{\text {nom }, i}}{\sigma_{i}} \frac{f_{i}-f_{n o m, i}}{f_{\text {nom }, i}} \\
P_{l i} & =P_{l i}^{o} \cdot\left(1+D_{l i}\left(f_{i}-f_{\text {nom }, i}\right)\right) .
\end{aligned}
$$

The frequency $f_{i}(t)$ can be readily measured and hence used for feedback control. Its evolution (1) expresses a balance between generated power, consumed power and angular acceleration. Equation (2) expresses local primary frequency control, i.e. the standard adjustment of mechanical power input $P_{m i}(t)$ to counter deviations from nominal frequency $f_{n o m, i}$ within the $\mathrm{AC}$ area. $J_{i}$ is 
the moment of inertia of the aggregated area $i$ generator and $D_{g i}$ its damping factor; $\sigma_{i}$ is called the generator droop, $P_{n o m, i}$ its rated mechanical power, $T_{s m i}$ the time constant for local power adjustment. The reference power $P_{m i}^{o}$ is adapted by secondary frequency control over timescales no shorter than $30 \mathrm{~s}$, and can therefore be considered as a constant parameter $P_{m i}^{o}=\bar{P}_{m i}^{o}$ for our much faster primary frequency control. The aggregated power load $P_{l i}(t)$ fluctuates with sensitivity factor $D_{l i}$ as a function of frequency, see (3). Its nominalfrequency value $P_{l i}^{o}(t)$ can be viewed as an input of the consumers to the power network. The power $P_{i}^{d c}(t)$ injected by area $i$ into the DC grid through its converter governs its interaction with the HVDC network.

Converters are complex nonlinear dynamical systems. At the timescales of our algorithm, they can be modeled as devices that consume no power between AC and DC side, and capable of instantaneously applying either a given DC-side voltage $V_{i}^{d c}(t)$ or a given power injection $P_{i}^{d c}(t)$. We will thus alternately consider either the $V_{i}^{d c}$ or the $P_{i}^{d c}$ as actuated variables. The DC sides of converters $i$ and $k$ are connected in the DC grid through a resistance $R_{i k}$. If converters $i, k$ are not connected, we assign $R_{i k}=\infty$. Then by Ohm's law, the power flows $P_{i}^{d c}(t)$, for $i=1,2, \ldots, N$, satisfy

$$
P_{i}^{d c}=V_{i}^{d c} \sum_{k=1}^{N} \frac{\left(V_{i}^{d c}-V_{k}^{d c}\right)}{R_{i k}} .
$$

We put a bar over a symbol to indicate its value at the reference operating point. The latter is a particular equilibrium at which the system is assumed to rest in the absence of disturbances. It has all frequencies equal to their nominal values $\bar{f}_{i}=f_{n o m, i}$ and further depends on some reference powers and voltages. In practice, fixing the reference operating point is a complex process that may involve several actors. Equations (1),(2) at equilibrium and (3),(4) impose among others: $\bar{P}_{m i}=\bar{P}_{m i}^{o}$ and $\bar{P}_{l i}=\bar{P}_{l i}^{o}=\bar{P}_{m i}^{o}-\bar{P}_{i}^{d c}, \forall i$.

The range in which $P_{m i}(t)$ can vary around $P_{m i}^{o}$ by following (2) is called the primary reserve of area $i$. Providing sufficient primary reserves entails non-negligible costs to transmission system operators [13]; e.g. the procurement costs in primary reserve markets in Germany totaled around $€ 80$ million in 2006 [17]. The aim in the present paper is to propose converter control algorithms that make the whole HVDC network cooperatively react to any imbalances. The $\mathrm{AC}$ areas then 'share their primary reserves', so the necessary reserve in each individual area might be downsized. Since primary frequency control is designed for power imbalances that are relatively small with respect to the total generation capacity ${ }^{1}$, we consider small deviations from the reference operating point. We define:

\footnotetext{
${ }^{1}$ In case of larger imbalances, that are pretty rare, emer-
}

$$
\begin{array}{ll}
\text { states: } & x_{i}=P_{m i}-\bar{P}_{m i}^{o} \\
& y_{i}=f_{i}-f_{n o m, i} \quad(=\text { output }) ; \\
\text { disturbance: } & d_{i}=P_{l i}^{o}-\bar{P}_{l i}^{o} ; \\
\text { control input: } & u_{i}=P_{i}^{d c}-\bar{P}_{i}^{d c} \text { or } \quad v_{i}=V_{i}^{d c}-\bar{V}_{i}^{d c}
\end{array}
$$

for $i=1,2, \ldots, N$. We denote $x$ the column-vector with components $x_{i}$, and similarly for $y, d, u$ and $v$. Table 1 gathers further notation. As the most hazardous event in primary frequency control is the instant loss of a generator group or a significant load, our analysis focuses on a step variation of $d_{i}$.

Table 1

Notation and parameters.

\begin{tabular}{|ll|}
\hline$D_{i}$ & $=D_{g i}+\bar{P}_{l i}^{o} D_{l i} /\left(4 \pi^{2} f_{n o m, i}\right)>0$ \\
$a_{1 i}$ & $=D_{i} / J_{i}>0$ \\
$a_{2 i}$ & $=1 /\left(4 \pi^{2} f_{n o m, i} J_{i}\right)>0$ \\
$a_{3 i}$ & $=P_{n o m, i} /\left(T_{s m i} \sigma_{i} f_{n o m, i}\right)>0$ \\
$a_{4 i}$ & $=1 / T_{s m i}>0$ \\
$m_{1 i}$ & $=a_{1 i} / a_{2 i}+a_{3 i} / a_{4 i}>0$ \\
$m_{2 i}$ & $=P_{n o m, i} /\left(\sigma_{i} f_{n o m, i}\right)=a_{3 i} / a_{4 i}>0$ \\
$A_{k}, M_{k}$ & diagonal matrix of the $a_{k i}$ resp. $m_{k i}$ \\
$\bar{V}, \bar{C}$ & diagonal matrix of the $\bar{V}_{i}^{d c}$ resp. $\bar{P}_{i}^{d c}$ \\
0 & all-zero matrices of appropriate sizes \\
$I$ & identity matrix $\in \mathbb{R}^{N \times N}$ \\
$q^{*}$ & complex conjugate transpose of $q$ \\
\hline
\end{tabular}

\section{Power-injection-based control scheme}

If all the converters try to independently impose their power injection $P_{i}^{d c}$, a severe HVDC power balance conflict can result, indicated by the fact that (4) has no (realistic) solution. We therefore apply power-injection control to the first $N-1$ converters only, and compute a compatible value of $P_{N}^{d c}$ for fixed $V_{N}^{d c}=V_{r e f}^{d c}$.

Primary reserve sharing means collective reaction to disturbances. We therefore want to design the $u_{i}(t)$ such that the $y_{i}$ all tend to be equal. Driving variables to a common value is addressed by consensus algorithms (see e.g. the review [11]): area $i$ would control its dynamics to drive $y_{i}$ towards the average of output values $\left\{y_{k}\right\}$ of some other areas $\{k\}$, known through communication. Originally developed for simple integrators [21], consensus has been generalized to other situations including linear second-order systems [16]. Following this line of work, we let proportional-integral type subcontrollers

gency control strategies such as load-shedding actions usually supersede primary frequency control. 


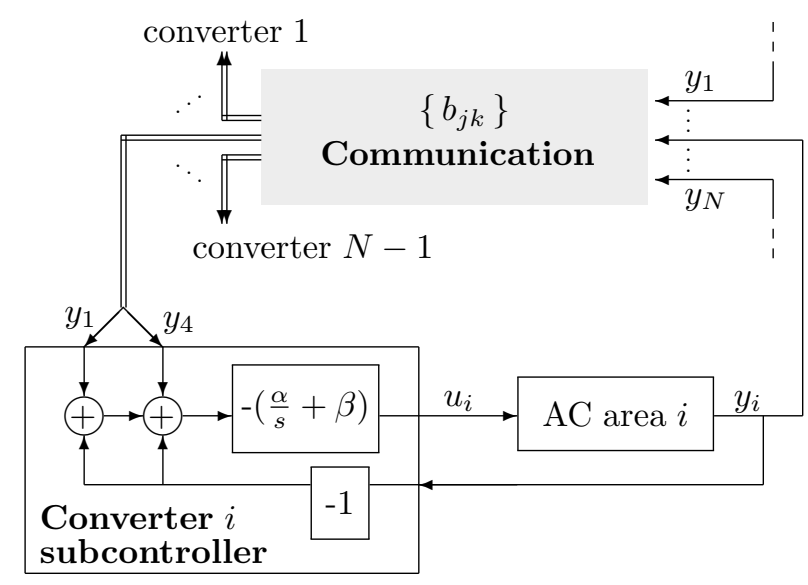

Fig. 2. Schematic representation of the power-injection-based controller. Double lines denote communication channels carrying several variables. The area $i$ example subcontroller receives frequency information from areas 1 and 4 . A similar subcontroller structure is applied for all $i=1,2, \ldots, N-1$. Cooperative frequency control relies on local subcontrollers and explicit communication, shown in bold.

drive $y_{i}$ towards the $y_{k}$ for $i \in\{1,2, \ldots, N-1\}$ :

$$
u_{i}=\sum_{k=1}^{N} b_{i k}\left(\alpha \int\left(y_{i}-y_{k}\right) d t+\beta\left(y_{i}-y_{k}\right)\right)
$$

where $\alpha, \beta$ are positive gains and the coefficients $b_{i k}$ model communication: $b_{i k}=1$ if subcontroller $i$ receives frequency information from area $k$, otherwise $b_{i k}=0$. One could more generally tune gains $\alpha_{i k}, \beta_{i k}$ as a function of areas $i$ and $k$, but this goes beyond the scope of the present paper. Figure 2 illustrates the system with this controller. The HVDC grid does not explicitly show up: its only role is to physically allow the $N-1$ converters to transfer power to somewhere. The different behavior of converter $N$ is a departure from classical consensus. However, in practice it turns out that $u_{N}(t)$ follows the same dynamics (5) in good approximation (see next).

We study the closed-loop system linearized around the reference operating point. Numerical simulations further explore the full nonlinear model in Section 5. We make the following assumptions.

Assumption 1 The graph representing frequency deviation communication among the subcontrollers is

- constant in time;

- undirected: $b_{i k}=b_{k i} \forall i, k$, that is if subcontroller $k$ has access to $y_{i}$ then subcontroller $i$ has access to $y_{k}$;

- connected: for each pair of agents $i, j$ there exists a set of indices $k_{1}, k_{2}, \ldots, k_{m}$ such that $k_{1}=i, k_{m}=j$ and $b_{k_{n} k_{n+1}}=1$ for $n=1,2, \ldots, m-1$.

Assumption 2 The variation of the net overall power flow injected into the DC grid can be neglected, i.e. $\sum_{i=1}^{N} u_{i}=0$.
Assumption 2 simplifies (4), which rigorously imposes $\sum_{i=1}^{N}\left(P_{i}^{d c} / V_{i}^{d c}-\bar{P}_{i}^{d c} / \bar{V}_{i}^{d c}\right)=0$ with the $V_{i}^{d c}$ to be computed from nonlinear coupled equations. The approximation holds because relative variations of the exchanged power largely exceed relative variations of the voltage in practice. The conclusions obtained under this approximation agree with simulations of the exact model in Section 5. Assumptions 1 and 2 imply that $u_{N}$ also satisfies (5), with $b_{N i}=b_{i N}$ for $i=1,2, \ldots, N$.

The only nonlinear differential equation, resulting from (1) and (3), has the standard linearization

$$
J_{i} \frac{d}{d t} y_{i}=\frac{x_{i}-d_{i}-u_{i}}{4 \pi^{2} f_{\text {nom }, i}}-D_{i} y_{i} .
$$

The linearized closed-loop system is then given by electromechanical dynamics (6) and primary frequency control (2) in the individual areas, plus our HVDC powerinjection controller $(5)$, for all areas $i=1,2, \ldots, N$. Denote $L \in \mathbb{R}^{N \times N}$ the Laplacian matrix of the graph describing inter-area communication, whose off-diagonal elements are $l_{i, k}=-b_{i k} \forall k, i \neq k$ and diagonal elements $l_{i, i}=\sum_{k} b_{i k}$. Then the closed-loop system writes:

$$
\begin{aligned}
& \frac{d}{d t}\left(\begin{array}{l}
y \\
x \\
u
\end{array}\right)=S\left(\begin{array}{l}
y \\
x \\
u
\end{array}\right)-\left(\begin{array}{l}
A_{2} \\
0 \\
0
\end{array}\right) d \\
& \text { with } \quad S=\left(\begin{array}{ccc}
-A_{1} & A_{2} & -A_{2} \\
-A_{3} & -A_{4} & 0 \\
L\left(\alpha I-\beta A_{1}\right) & \beta L A_{2} & -\beta L A_{2}
\end{array}\right)
\end{aligned}
$$

Proposition 3 Consider the system (2), (5), (6) satisfying Assumptions 1 and 2. Then at the equilibrium point associated to load imbalances $\left\{d_{i}: i=1,2, \ldots, N\right\}$, the frequency deviations of all areas are equal and given by

$$
y_{i}=-\left(\sum_{k} d_{k}\right) /\left(\sum_{k} m_{1 k}\right) \quad \forall i \in\{1,2, \ldots, N\} .
$$

Mechanical power correspondingly varies as

$$
x_{i}=-m_{2 i} y_{i}
$$

Proof: Annihilating the derivative of (5) under Assumption 1 imposes $y_{i}=y_{k}=: y^{e}$ for all $i, k$, with $y^{e}$ unspecified. (This is indeed the unique zero-eigenvector of the Laplacian associated to the communication graph — standard consensus argument, see e.g. $[11,16]$.) Then imposing equilibrium in (6),(2) expresses $x_{i}$ and $u_{i}$ as a function of $d_{i}$ and $y^{e}$. The conservation of $\sum_{i=1}^{N} u_{i}$ from Assumption 2 finally fixes the value of $y^{e}$.

The sharing of a load disturbance in equal frequency deviations for all $\mathrm{AC}$ areas is reminiscent of an all-AC 
network. The $x_{i}$ values further reflect primary reserve sharing. Consider for instance an imbalance $d_{i}=\bar{d}$ in AC area $i$ and $d_{k}=0 \forall k \neq i$. Keeping $u_{k}=0 \forall k$ would then yield $x_{i}=\left(m_{2 i} / m_{1 i}\right) \bar{d}$ and $x_{k}=0 \forall k \neq i$. With our controller, $x_{i}$ is reduced by a factor $\left(\sum_{k} m_{1 k}\right) / m_{1 i}$, while the other areas contribute similarly. This reduces $\max _{k}\left(x_{k}\right)$, hence the necessary primary reserve in individual areas. $\mathrm{AC}$ areas with larger $m_{2 i}$ - giving more weight to frequency deviations in their local controller (2) - contribute more to the collective reaction.

Proposition 4 The system (7) restricted to the subspace $\sum_{i=1}^{N} u_{i}=0$ is stable for any communication graph satisfying Assumption 1 , for any $\alpha>0$ and $\beta \geq 0$.

Proof: The restriction to the subspace cancels one 0 eigenvalue of $S$ corresponding to a continuum of equilibria (see also Proposition 3 ). If the 0 eigenvalue had algebraic multiplicity $\geq 2$ for $S$, then it would have geometric multiplicity $\geq 2$ for $S^{2}$. One checks by rank-preserving transformations that $S^{2}$ has rank $3 N-1$, confirming that 0 is an eigenvalue of algebraic multiplicity 1 for $S$, when $\alpha \neq 0$. It thus remains to show that $S$ can have no eigenvalue $\lambda \neq 0$ with $\Re e(\lambda) \geq 0$.

By contradiction, assume that $S$ has an eigenvalue $\lambda \neq 0$ with $\Re e(\lambda) \geq 0$, associated to an eigenvector with $y=$ $q_{1} \in \mathbb{C}^{N}, x=q_{2} \in \mathbb{C}^{N}, u=q_{3} \in \mathbb{C}^{N}$. A few algebraic operations with (7) lead to the conditions:

$q_{3}=(\beta+\alpha / \lambda) L q_{1}$

$q_{3}=-\left(\frac{\lambda I+A_{1}}{A_{2}}+\frac{A_{3}}{\lambda I+A_{4}}\right) q_{1}$.

Note that $q_{1}=0$ would imply $q_{2}=q_{3}=0$, so we must have $q_{1} \neq 0$. Since the Laplacian of an undirected graph is positive semi-definite, (10) then requires $\Re e\left(q_{1}^{*} q_{3}\right) \geq 0$ and (11) requires $\Re e\left(q_{1}^{*} q_{3}\right)<0$. There can thus be no eigenvector with an eigenvalue $\lambda \neq 0, \Re e(\lambda) \geq 0$.

Decentralized control methods often assume identical subsystems. The 'consensus' literature [11] moreover considers simple subsystem dynamics to focus on switching graphs, which covers robotic and computer networks applications. For power networks the situation is opposite: under normal operation they undergo little qualitative changes, but assuming identical AC areas is unrealistic. This motivates our non-standard proof.

In a practical implementation of (5), the explicit communication of remote information between the AC areas introduces delays. We show in [3] that the system can be destabilized for time delays of a few hundred milliseconds. This motivates the proposal of a second control scheme, that does not rely on explicit communication.

\section{DC-voltage-based control scheme}

When actuation takes place through DC-side voltage $V_{i}^{d c}$, all $N$ converters can independently set their $v_{i}$ val-

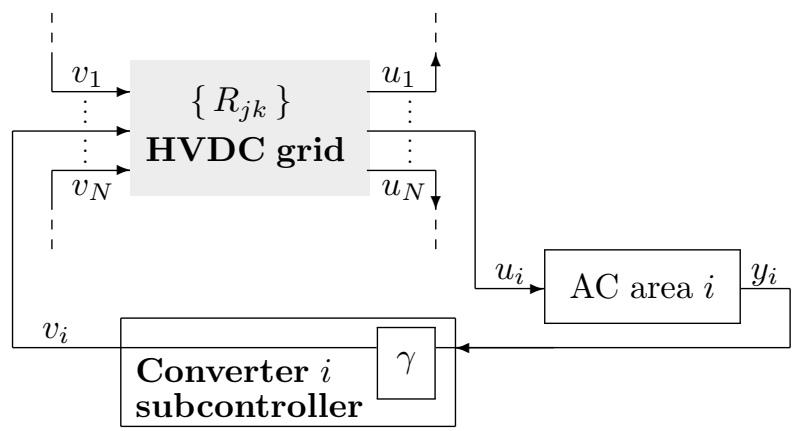

Fig. 3. Schematic representation of the voltage-based controller. It relies on local subcontrollers and on the constitutive physical law (4) of the HVDC grid, both shown in bold.

ues without creating a conflict.

The goal is to dispose of explicit communication, i.e. regulating $v_{i}$ as a function of $y_{i}$ only. Interaction then relies on the fact that $u_{i}$, an input variable to the dynamics (1)-(3) of AC area $i$, depends on the $v_{k}$ values set by other converters for which $R_{i k} \neq \infty$. Coordination thus explicitly relies on the plant-induced coupling. This approach differs from typical cooperative robotics as in $[6,11,16]$. To design a cooperative reaction, we note that a decreased frequency $y_{i}<0$ corresponds to a lack of power in $\mathrm{AC}$ area $i$; we should then increase power flow into AC area $i$, which happens if we decrease the voltage at its converter node. This motivates the control law

$$
v_{i}=\gamma y_{i} \quad, \text { for } i \in\{1,2, \ldots, N\}
$$

with positive gain $\gamma>0$. (All conclusions can be generalized to AC-area-dependent gains $\gamma_{i}$.) Figure 3 illustrates the system with this controller. The HVDC grid explicitly shows up to couple AC areas, unlike on Figure 2 . The subcontrollers provide inputs $v_{i}$ to this physical network where the laws of electricity, letting power flow as a function of voltage differences, essentially perform a frequency comparison to provide inputs $u_{i}$ to the $\mathrm{AC}$ areas. The $\left\{R_{i k}\right\}$ determine the interaction topology.

Similarly to Section 3, we study the closed-loop system linearized around the reference operating point. We must now explicitly consider the relation between the voltage and the power injection. Linearizing (4) yields:

$$
u_{i}=\frac{\bar{P}_{i}^{d c}}{\bar{V}_{i}^{d c}} v_{i}+\sum_{k=1}^{N} \frac{\bar{V}_{i}^{d c}}{R_{i k}}\left(v_{i}-v_{k}\right) .
$$

The second term in (13) is dominant for typical parameter values. Together with (12), it implies power injections that reflect differences among connected AC areas' frequency deviations, similarly to the control law of Section 3. We can therefore expect a similar consensus-like behavior among the frequencies. The first term in (13) together with (12), makes $u_{i}$ depend directly on $y_{i}$, unlike in our first controller. This difference would lead to 
an unstable system if we replaced the proportional controller (12) by a proportional-integral controller as used in (5). The following proves that (12) can yield a stable primary reserve sharing situation.

Denote $L^{R} \in \mathbb{R}^{N \times N}$ the weighted Laplacian matrix of the graph describing the HVDC grid, with off-diagonal elements $l_{i, k}^{R}=-1 / R_{i k} \forall k, i \neq k$ and diagonal elements $l_{i, i}^{R}=\sum_{k} 1 / R_{i k}$. The closed-loop system then writes:

$$
\begin{aligned}
& \frac{d}{d t}\left(\begin{array}{l}
y \\
x
\end{array}\right)=S^{\prime}\left(\begin{array}{l}
y \\
x
\end{array}\right)-\left(\begin{array}{l}
A_{2} \\
0
\end{array}\right) d \\
& \text { with } \quad S^{\prime}=\left(\begin{array}{cc}
-\left(A_{1}+\gamma A_{2}\left(\bar{C}+\bar{V} L^{R}\right)\right) & A_{2} \\
-A_{3} & -A_{4}
\end{array}\right) .
\end{aligned}
$$

Proposition 5 Consider the closed-loop system (14). If $\gamma$ is small enough such that $m_{1 i}+\gamma \bar{P}_{i}^{d c} / \bar{V}_{i}^{d c}>0$ for all $i$, then a load imbalance $d$ defines an equilibrium

$$
\begin{aligned}
& y=-\left(M_{1}+\gamma \bar{C}+\gamma \bar{V} L^{R}\right)^{-1} d \\
& x=-M_{2} y .
\end{aligned}
$$

In particular, for a load imbalance affecting area $i$ only, i.e. $d_{i}=\bar{d}>0$ and $d_{k}=0 \forall i \neq k$, this implies:

(a) $y_{k}<0$ for all areas in the connected DC grid.

(b) Area $i$ has the maximal deviation $\left|y_{i}\right|>\left|y_{k}\right| \forall k \neq i$. (c) Define $D=\bar{V}^{-1}\left(M_{1}+\gamma \bar{C}\right)$ diagonal, $G=$ $D^{-1 / 2} L^{R} D^{-1 / 2}$, and $G_{r} \in \mathbb{R}^{(N-1) \times(N-1)}$ by dropping row $i$ and column $i$ from $G$. Then

$$
\left|y_{i}\right|=\frac{\bar{d}}{m_{1 i}+\gamma \bar{P}_{i}^{d c} / \bar{V}_{i}^{d c}} \prod_{k=1}^{N-1} \frac{\left(1+\gamma \mu_{k}\right)}{\left(1+\gamma \lambda_{k}\right)}
$$

where the $(N-1)$ largest eigenvalues $\lambda_{k}$ of $G$ and eigenvalues $\mu_{k}$ of $G_{r}$ satisfy

$$
\lambda_{1} \geq \mu_{1} \geq \lambda_{2} \geq \mu_{2} \geq \ldots \geq \lambda_{N-1} \geq \mu_{N-1} \geq 0 .
$$

Proof: Equilibrium conditions for (14) directly yield (15),(16), with $m_{1 i}+\gamma \bar{P}_{i}^{d c} / \bar{V}_{i}^{d c}>0$ guaranteeing invertibility: for instance, applying the Gershgorin circle criterion on the rows of $Q:=\left(M_{1}+\gamma \bar{C}+\gamma \bar{V} L^{R}\right)$ guarantees that its eigenvalues have strictly positive real part. Now we analyze (15) for the particular imbalance affecting area $i$ only. If $y_{1}, y_{2}, \ldots, y_{N}$ are not all equal, then in the connected HVDC network there exists an area $k$ such that $y_{k} \geq y_{j} \forall j$ and $y_{k}>y_{l}$ for at least one area $l$ connected to area $k$. We then have $\sum_{j} \frac{1}{R_{k j}}\left(y_{k}-y_{j}\right)>0$. Line $k$ of $Q y=-d$ therefore requires $\left(m_{1 k}+\gamma \frac{\bar{P}_{k}^{d c}}{\bar{V}_{k}^{d c}}\right) y_{k}<-d_{k} \leq 0$. If in contrast $y_{1}=y_{2}=\ldots=y_{N}$, then line $i$ of $Q y=-d$ gives $\left(y_{1}=y_{2}=\ldots=\right) y_{i}=-\bar{d} /\left(m_{1 i}+\gamma_{\bar{P}_{i}^{d c}}\right)<0$. Thus (a) holds in both cases. Now assume that there exists an area $k \neq i$ such that $\left|y_{k}\right| \geq\left|y_{j}\right| \forall j$, contradicting (b). Then $y_{k}<0$ and $\left(y_{k}-y_{j}\right) \leq 0 \forall j$, but line $k$ of $Q y=-d$ requires $\left(m_{1 k}+\gamma \frac{\bar{P}_{k}^{d c}}{\bar{V}_{k}^{d c}}\right) y_{k}+\gamma \bar{V}_{k}^{d c} \sum_{j} \frac{1}{R_{k j}}\left(y_{k}-y_{j}\right)=0$. This leads to a contradiction, so (b) must be true. For (c), first note that $Q y=-d$ indeed writes $\left(D+\gamma L^{R}\right) y=-\frac{1}{V_{i}^{d c}} d$. Solving this for $y_{i}$ with the Schur complement method yields $y_{i}=-\frac{\operatorname{det}\left(D_{r}+\gamma L_{r}^{R}\right)}{\operatorname{det}\left(D+\gamma L^{R}\right)} \frac{\bar{d}}{\bar{V}_{i}^{d c}}$, where $D_{r}, L_{r}^{R} \in \mathbb{R}^{(N-1) \times(N-1)}$ are obtained by dropping row $i$ and column $i$ from $D$ and $L^{R}$ respectively. Now observe that $\operatorname{det}\left(D+\gamma L^{R}\right)=\operatorname{det}\left(D^{1 / 2}(I+\right.$ $\left.\left.\gamma D^{-1 / 2} L^{R} D^{-1 / 2}\right) D^{1 / 2}\right)=\operatorname{det}(D) \operatorname{det}(I+\gamma G)$ and the same with ${ }_{r}$ indices. Diagonality of $D$ ensures that $G_{r}$ is as well obtained by dropping row $i$ and column $i$ from $G$. Formula (17) follows by writing out the determinants in eigenvalues. The "eigenvalue interleaving" in (18) is a standard property of a symmetric matrix and one of its $(N-1) \times(N-1)$ principal sub-matrices.

Property (a) shows that the AC areas collectively react to any local load imbalance. In absence of integral action, power flows are directly driven by frequency differences, so it is unavoidable that the $y_{i}$ of different $\mathrm{AC}$ areas take different values at equilibrium (15). For realistic parameter values, $m_{1 i}$ dominates $\gamma\left|\bar{P}_{i}^{d c} / \bar{V}_{i}^{d c}\right|$. Then the product of $\left(1+\gamma \mu_{k}\right) /\left(1+\gamma \lambda_{k}\right)$ in (17) characterizes how much the maximal frequency deviation following a local imbalance is reduced thanks to our controller. Power transfers are obviously not expected to help when all areas undergo similar load disturbances. For that case, (15) says that our controller might even lead to (typically slightly) increased maximal deviations.

Proposition 6 If $\gamma>0$ is small enough such that $4 \pi^{2} f_{\text {nom }, i} D_{i}+\gamma \frac{\bar{P}_{i}^{d c}}{\bar{V}_{i}^{d c}}>0$ for all $i$, then the equilibrium in Proposition 5 is asymptotically stable.

Proof: Write $y=q_{1}, x=\bar{V} q_{2}$ a potential eigenvector of $S^{\prime}$ associated to an eigenvalue $\lambda$ with $\Re e(\lambda) \geq 0$, to find a contradiction similarly to Proposition 4.

The assumptions involving $\gamma$ in Propositions 5 and 6 are just sufficient bounds. We have found unstable systems with $m_{1 i}+\gamma \bar{P}_{i}^{d c} / \bar{V}_{i}^{d c}>0$ though, confirming that the tighter bound for Proposition 6 is justified. Both bounds can be checked locally in each AC area, without requiring any knowledge about other areas or the network topology $\left(L^{R}\right)$. On the other hand, since the parameters of a power system are essentially invariant in time, a central system designer with knowledge of all the HVDC network parameters could perform a more detailed analysis, e.g. assessing stability from (14) directly. He could then fine-tune the controller parameters offline and send them to the local operators, prior to online operation with decentralized dynamic variables $x_{i}, y_{i}$ and $v_{i}$. The important economic context could still motivate decentralized 
Table 2

Parameter values for the simulated system.

\begin{tabular}{|c|c|c|c|c|c|c|}
\hline \multicolumn{6}{|c|}{ HVDC grid } & \multirow[t]{2}{*}{ Unit } \\
\hline$R_{12}$ & $R_{15}$ & $R_{23}$ & $R_{25}$ & $R_{34}$ & $R_{45}$ & \\
\hline 1.39 & 4.17 & 2.78 & 6.95 & 2.78 & 2.78 & $\Omega$ \\
\hline $\mathrm{AC}$ areas & 1 & 2 & 3 & 4 & 5 & \\
\hline$J$ & 2026 & 6485 & 6078 & 2432 & 4863 & $\mathrm{~kg} \mathrm{~m}^{2}$ \\
\hline$D_{g}$ & 48.4 & 146.4 & 140 & 54.7 & 95 & $\mathrm{~W} \mathrm{~s}{ }^{2}$ \\
\hline$T_{s m}$ & 1.5 & 2.0 & 2.5 & 2 & 1.8 & $\mathrm{~s}$ \\
\hline$\sigma$ & 0.02 & 0.04 & 0.06 & 0.04 & 0.03 & / \\
\hline$P_{\text {nom }}$ & 50 & 80 & 50 & 30 & 80.4 & MW \\
\hline $\bar{V}^{d c}$ & 99.17 & 99.60 & 99.73 & 99.59 & 100 & $\mathrm{kV}$ \\
\hline $\bar{P}^{d c}$ & -50 & 20 & 10 & -20 & 40.4 & MW \\
\hline $\bar{P}_{l}^{o}$ & 100 & 60 & 40 & 50 & 40 & MW \\
\hline \multicolumn{7}{|c|}{$f_{\text {nom }, i}=50 \mathrm{~Hz}, D_{l i}=0.1 \mathrm{~s}$ and $\bar{P}_{m, i}^{o}=P_{n o m, i}$ for all $i$} \\
\hline
\end{tabular}

tuning strategies, where local agents do not communicate their area characteristics to any central authority. If the reference operating point uses zero power exchanges $\bar{P}_{i}^{d c}=0 \forall i$, meaning $\bar{V}_{i}^{d c}=\bar{V}_{k}^{d c} \forall i, k$, then (and only then) $\bar{C}$ has no negative components. Propositions $5 \&$ 6 then allow arbitrarily large $\gamma$ and larger $\gamma$ always yield smaller $\left|y_{i}\right|$. These are however associated to larger $V_{i}^{d c}$ and $P_{i}^{d c}$ variations, which limits the gain in practice.

\section{Simulations}

We illustrate the two control schemes on the 5-terminal HVDC system of Fig. 1, modeled as a purely resistive grid with realistic parameter and reference operating point values given in Table 2 . We simulate the full nonlinear model (1)-(4). To observe the system's response to a power imbalance, we assume that all areas initially operate at the reference operating point and we increase $P_{l 2}^{o}$ by $5 \%$ at time $t=2 \mathrm{~s}$. The ensuing evolution of $f_{2}$ without primary reserve sharing - i.e. keeping $P_{i}^{d c}=\bar{P}_{i}^{d c}$ and $V_{i}^{d c}=\bar{V}_{i}^{d c}$ for all $i$ is drawn with blue circles on Figures 4 and 5 . It features a maximal transient deviation of $0.196 \mathrm{~Hz}$ and stabilizes at $49.927 \mathrm{~Hz}$, which matches the deviation given by (15) with $\gamma=0$; this validates our linearization for the theoretical study. The primary reserve usage $P_{m 2}-\bar{P}_{m 2}^{o}=x_{2}$ equals $2.93 \mathrm{MW}$. For other areas obviously $y_{i}=x_{i}=0$.

Now we apply our power-injection-based control (5) for $i=1,2,3,4$, fix $V_{5}^{d c}=100 \mathrm{kV}$ and compute all other converter variables to ensure perfect consistency of (4). Our communication graph coincides with the DC network topology. Figure 4 shows the frequency evolutions for $\alpha=\beta=2 \times 10^{6}$, assuming instantaneous communication. The maximal transient deviation of $f_{2}$ is $0.136 \mathrm{~Hz}$ and the frequencies of all areas converge to each other to settle at $49.983 \mathrm{~Hz}$, matching (8). The primary reserve consumptions at steady state range from $x_{4}=251 \mathrm{~kW}$

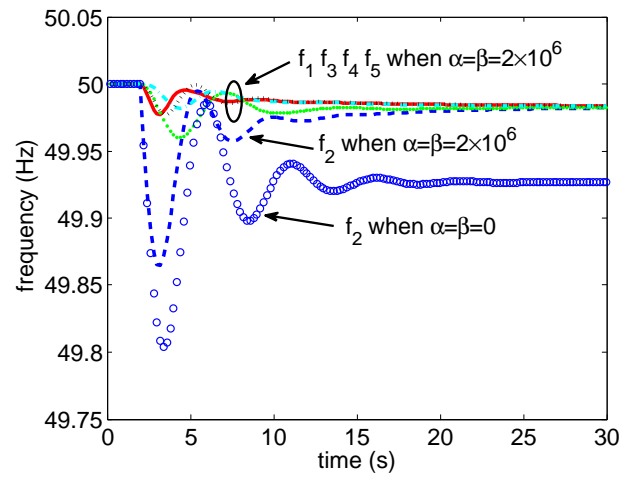

Fig. 4. Frequencies of the five $\mathrm{AC}$ areas under the power-injection-based control scheme with $\alpha=\beta=2 \times 10^{6}$. Blue circles also show the evolution of $f_{2}$ when $\alpha=\beta=0$.

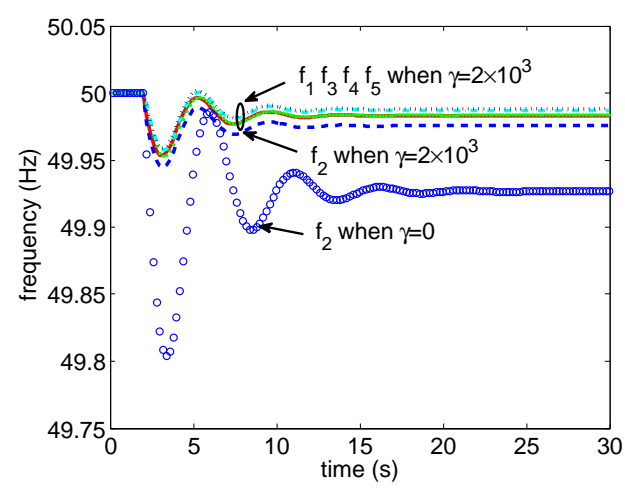

Fig. 5. Frequencies of the five AC areas under the DC-voltage-based control scheme with $\gamma=2 \times 10^{3}$. Blue circles also show the evolution of $f_{2}$ when $\gamma=0$.

to $x_{5}=898 \mathrm{~kW}$. The maximal effort by an individual $\mathrm{AC}$ area is thus divided by about 3. Larger controller gains reduce the transient deviation of $f_{2}$. This is limited in practice among others by time delays. With the chosen $\alpha=\beta=2 \times 10^{6}$, communication delays above $0.57 \mathrm{~s}$ can destabilize the system.

Next we apply the DC-voltage-based control (12). We take $\gamma=2 \times 10^{3}$ to meet the conditions of Propositions 5 and 6 . Figure 5 shows how the frequencies follow similar variations to finally settle between $49.976 \mathrm{~Hz}$ and $49.988 \mathrm{~Hz}$, in agreement with (15). The $0.024 \mathrm{~Hz}$ steadystate deviation of $f_{2}$ is larger than with the first controller, but still three times less than without control. Primary reserve consumption $x_{i}$ at steady state reduces to $976 \mathrm{~kW}$ for AC area 2 and lies between 194 and $843 \mathrm{~kW}$ for the other areas. The $0.055 \mathrm{~Hz}$ maximum transient deviation of $f_{2}$ is lower than with the first controller. For $\gamma=4 \times 10^{3}$, the frequencies settle within a smaller bandwidth of $0.007 \mathrm{~Hz}$ and $\mathrm{AC}$ area 1 contributes more than AC area $2\left(x_{1}=850 \mathrm{~kW}, x_{2}=837 \mathrm{~kW}\right)$. Although this larger $\gamma$ violates the condition for Proposition 6, an eigenvalue computation shows that $S^{\prime}$ is still stable. 
The $V_{i}^{d c}$ (resp. $P_{i}^{d c}$ ) vary by no more than $0.3 \%$ (resp. 12\%) in the above simulations, with instantaneous variations within modern converters' tracking speeds (see e.g. $[10,12]$ ). We have also simulated our controllers with $P_{l i}^{o}$ variations up to $50 \%$ in one $\mathrm{AC}$ area, without observing instabilities.

\section{Conclusion}

We have presented controllers that make an HVDC system collectively react to local variations in the (handily measurable) AC area frequencies. A first scheme, requiring communication, regulates the power injection from each AC area into the DC grid as a function of compared frequency deviations in neighboring areas, much like a standard consensus algorithm. A second control scheme just sets the DC-side voltage of each converter proportionally to its local frequency deviation. Each AC area thereby "signals" its imbalance through the natural dynamics of the DC grid so that coordination is achieved without explicit communication. Theoretical analysis proves that the interconnected system locally converges to a stable equilibrium with each controller. The frequency deviations and primary reserve consumptions resulting from a local power imbalance are shared between AC areas, such that the affected area's effort is significantly reduced. This analysis is valid for AC areas with different individual characteristics and confirmed by simulations. Primary reserves are thus shared like in an $\mathrm{AC}$ network, but with a controlled coupling that e.g. can easily accommodate firewalls to prevent cascading outages.

\section{References}

[1] S. Bhamidipati and A. Kumar. Load frequency control of an interconnected system with DC tie-lines and AC-DC parallel tie-lines. Proc. 22nd Annual North American Power Symp., 1:390, 1990.

[2] R. Billinton and N.A. Chowdhury. Operating reserve assessment in interconnected generating systems. IEEE Trans. on Power Systems, 3(4):1479-1487, 1988.

[3] J. Dai, Y. Phulpin, A. Sarlette, and D. Ernst. Impact of delays on a consensus-based primary frequency control scheme for AC systems connected by a multi-terminal HVDC grid. Proc. IREP Symp. on Bulk Power Systems Dynamics \& Control, 2010.

[4] J. Dai, Y. Phulpin, A. Sarlette, and D. Ernst. Voltage control in an HVDC system to share primary frequency reserves between non-synchronous areas. Proc. 17th Power Systems Computation Conf., 2011.

[5] J. Dai, Y. Phulpin, A. Sarlette, and D. Ernst. Coordinated primary frequency control among non-synchronous systems connected by a multi-terminal HVDC grid. IET Generation, Transmission \& Distribution, 6(2):99-108, 2012.

[6] J.A. Fax and R.M. Murray. Information flow and cooperative control of vehicle formations. IEEE Trans. on Automatic Control, 49(9):1465-1476, 2004.
[7] G. Fujita, G. Shirai, and R. Yokoyama. Automatic generation control for DC-link power system. IEEE/PES Transmission and Distribution Conf. \& Exhibit. : Asia Pacific, 3:15841588, 2002.

[8] P. Kundur. Power system stability and control. McGraw-Hill, 1994.

[9] Changsong Li, Y. Okada, M. Watanabe, and Y. Mitani. Modeling Kita-Hon HVDC link for load frequency control of Eastern Japan $50-\mathrm{Hz}$ power system based on application of the CampusWAMS. Proc. IEEE Int. Symp. on Circuits and Systems, pages 2307-2310, 2010.

[10] K. Meah and A.H.M. Sadrul Ula. A new simplified adaptive control scheme for multi-terminal HVDC transmission systems. Int.l J. of Electrical Power \& Energy Systems, 32(4):243-253, 2010.

[11] R. Olfati-Saber, J.A. Fax, and R.M. Murray. Consensus and cooperation in networked multi-agent systems. Proceedings of the IEEE, 95(1):215-233, 2007.

[12] K.R. Padiyar and N. Prabhu. Modelling, control design and analysis of VSC based HVDC transmission systems. pages 774-779, 2004.

[13] K.A. Papadogiannis and N.D. Hatziargyriou. Optimal allocation of primary reserve services in energy markets. IEEE Trans. on Power Systems, 19(1):652-659, 2004.

[14] N.S. Rau, C. Necsulescu, K.F. Schenk, and R.B. Misra. A method to evaluate economic benefits in interconnected systems. IEEE Trans. on Power Apparatus and Systems, 102(2):472-482, 1983.

[15] Y.G. Rebours, D.S. Kirschen, M. Trotignon, and S. Rossignol. A survey of frequency and voltage control ancillary services, part 1: technical features. IEEE Trans. on Power Systems, 22(1):350-357, 2007.

[16] W. Ren and R. Beard. Distributed consensus in multivehicle cooperative control: theory and applications. Springer London, 2008.

[17] S. Riedel and H. Weigt. German electricity reserve markets. Electricity Markets Working Paper, nr. EM-20.

[18] M. Sanpei, A. Kakehi, and H. Takeda. Application of multi-variable control for automatic frequency controller of HVDC transmission system. IEEE Trans. on Power Delivery, 2(9):1063-1068, 1994

[19] S. Sterpu and M.N. Tuan. Sharing frequency response between asynchronous electrical systems. IEEE Power \&6 Energy Society General Meeting, pages 1-6, 2009.

[20] Y. Tao, S. Shande, Z. Shouzhen, Z. Yuzhu, and Z. Weijiang. A novel auxiliary frequency controller for HVDC transmission links. Proc. Int. Conf. on Power System Technology, 1:515519, 2002.

[21] J.N. Tsitsiklis. Problems in decentralized decision making and computation. PhD Thesis, MIT, 1984. 